\title{
Incidence and Clinical Significance of Inducible Atrial Tachycardia in Patients with Atrioventricular Nodal Reentrant Tachycardia
}

\author{
CHRISTIAN STICHERLING, M.D., HIROSHI TADA, M.D., RADMIRA GREENSTEIN, M.D., \\ CHI-WO CHAN, M.D., STEVEN P. CHOUGH, M.D., ROBERT L. BAKER, M.D., \\ KRISTINA WASMER, M.D., HAKAN ORAL, M.D., FRANK PELOSI, M.D., \\ BRADLEY P. KNIGHT, M.D., S. ADAM STRICKBERGER, M.D., \\ and FRED MORADY, M.D.
}

From the Department of Internal Medicine, Division of Cardiology, University of Michigan Medical Center, Ann Arbor, Michigan

\begin{abstract}
Significance of Atrial Tachycardia. Introduction: The purpose of this prospective study was to determine the prevalence and clinical significance of inducible atrial tachycardia in patients undergoing slow pathway ablation for AV nodal reentrant tachycardia who did not have clinically documented episodes of atrial tachycardia.

Methods and Results: Twenty-seven (15\%) of 176 consecutive patients who underwent slow pathway ablation for AV nodal reentrant tachycardia were found to have inducible atrial tachycardia with a mean cycle length of $351 \pm 95$ msec. The atrial tachycardia was sustained in $7(26 \%)$ of 27 patients and was isoproterenol dependent in 20 patients $(\mathbf{7 4 \%})$. The atrial tachycardia was not ablated or treated with medications, and the patients were followed for $9.7 \pm 5.8$ months. Six $(22 \%)$ of the 27 patients experienced recurrent palpitations during follow-up. In one patient each, the palpitations were found to be due to sustained atrial tachycardia, nonsustained atrial tachycardia, recurrence of AV nodal reentrant tachycardia, paroxysmal atrial fibrillation, sinus tachycardia, and frequent atrial premature depolarizations. Thus, only $2(7 \%)$ of 27 patients with inducible atrial tachycardia later developed symptoms attributable to atrial tachycardia.

Conclusion: Atrial tachycardia may be induced by atrial pacing in $15 \%$ of patients with AV nodal reentrant tachycardia. Because the vast majority of patients do not experience symptomatic atrial tachycardia during follow-up, treatment for atrial tachycardia should be deferred and limited to the occasional patient who later develops symptomatic atrial tachycardia. ( $\mathrm{J}$ Cardiovasc Electrophysiol, Vol. 12, pp. 507-510, May 2001)
\end{abstract}

atrial tachycardia, paroxysmal supraventricular tachycardia, slow pathway

Introduction

During electrophysiologic testing in patients with AV nodal reentrant tachycardia, atrial tachycardia sometimes is induced by atrial pacing. In patients without clinically documented episodes of atrial tachycardia, the clinical significance of inducible atrial tachycardia may be uncertain. Therefore, it often is unclear whether treatment of the atrial tachycardia is necessary. The purpose of this prospective study was to determine the prevalence and clinical significance of atrial tachycardia induced in patients undergoing radiofrequency ablation for $\mathrm{AV}$ nodal reentrant tachycardia.

Supported in part by the Don Nouse Arrhythmia Research Fund. Dr. Sticherling was supported in part by the German Research Foundation.

Address for correspondence: Fred Morady, M.D., Department of Internal Medicine, Division of Cardiology, University of Michigan Medical Center, 1500 East Medical Center Drive, Box 0022, Ann Arbor, MI 49109-0022. Fax: 734-936-7026; E-mail: fmorady@ umich.edu

Manuscript received 18 December 2000; Accepted for publication 2 February 2001 .
Methods

\section{Characteristics of the Study Population}

The subjects of this study were 176 consecutive patients (130 women [74\%] and 46 men; mean age $48 \pm 17$ years) referred to the University of Michigan Medical Center for radiofrequency ablation of paroxysmal supraventricular tachycardia found to be caused by AV nodal reentrant tachycardia. None of these patients had prior ECG recordings suggestive of atrial tachycardia. Twenty-six patients $(15 \%)$ had underlying heart disease (coronary artery disease 15 , valvular heart disease 7 , dilated cardiomyopathy 3 , and hypertrophic cardiomyopathy 1 ). Mean left ventricular ejection fraction was $0.58 \pm 0.05$, and mean left atrial diameter was $36 \pm 7 \mathrm{~mm}$.

\section{Electrophysiologic Testing}

In all patients, antiarrhythmic drug therapy was discontinued at least five half-lives before the procedure. After obtaining informed consent and with the patients in a fasting state, three quadripolar electrode catheters were inserted into a femoral vein and positioned in the high right atrium, His-bundle position, and right ventricular apex. Several ECG leads and the intracardiac electrograms were displayed 


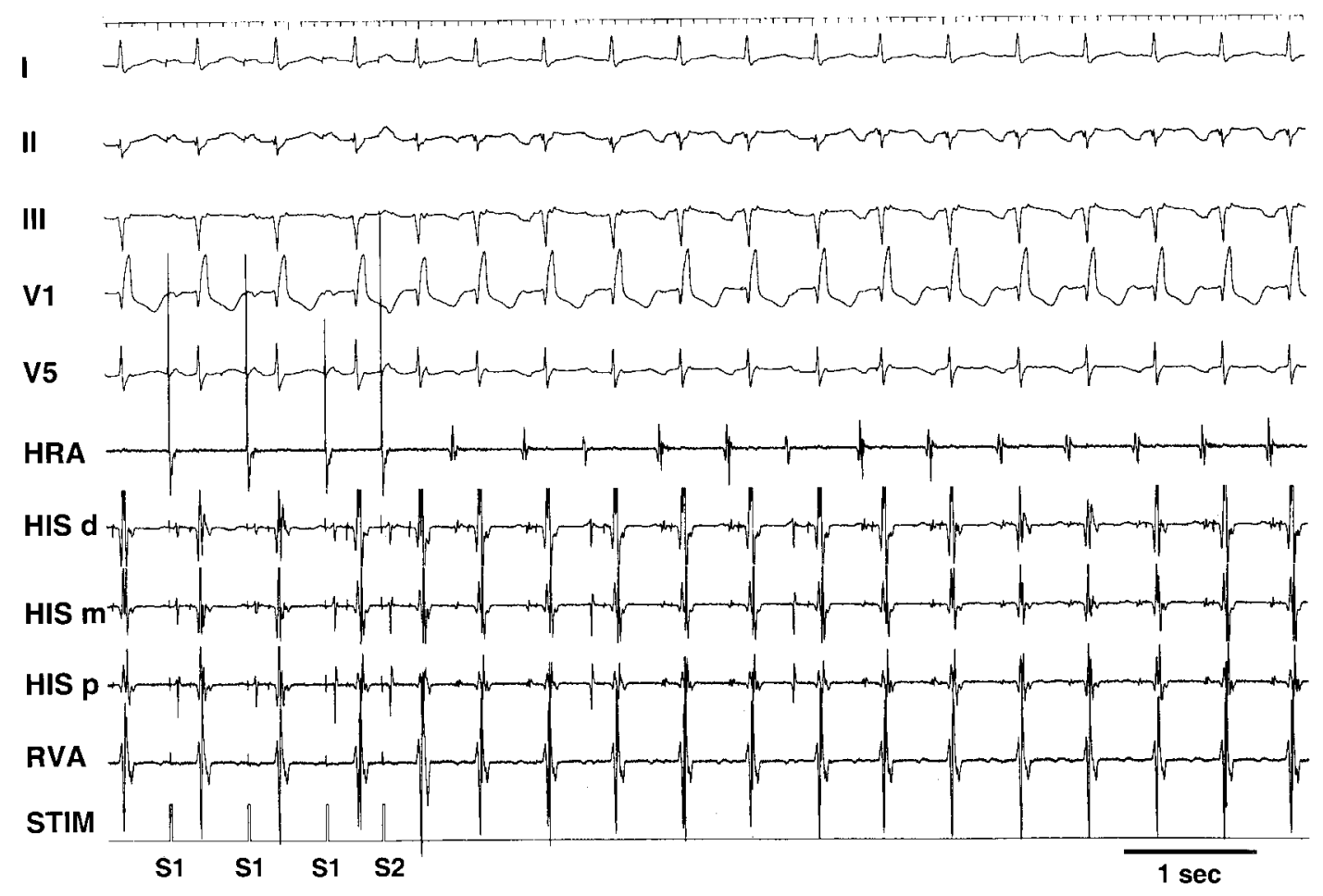

Figure 1. Example of sustained atrial tachycardia (cycle length $540 \mathrm{msec}$, duration $40 \mathrm{sec}$ ) induced by programmed atrial stimulation with a single extrastimulus. Displayed are leads I, II, III, $V_{1}$, and $V_{5}$, an electrogram recorded at the high right atrium (HRA), the distal (d), medial ( $m$ ), and proximal (p) His-bundle electrograms (HIS), right ventricular electrogram (RVA), and stimulus marker (STIM).

on an oscilloscope and recorded on optical disk (EPMedSystems, Mount Arlington, NJ, USA). Pacing was performed at twice the pacing threshold using a programmable stimulator (EPMedSystems).

\section{Study Protocol}

During the electrophysiologic procedure, incremental atrial and ventricular pacing and atrial and ventricular programmed stimulation with up to three extrastimuli were performed to induce tachycardia. If these maneuvers did not result in arrhythmia induction, they were repeated during infusion of isoproterenol 1 to $4 \mu \mathrm{g} / \mathrm{min}$. AV nodal reentrant tachycardia was induced in all patients. After slow pathway ablation, which was successful in all patients, the same pacing maneuvers were used to confirm successful elimination of the AV nodal reentrant tachycardia.

Because short episodes of inducible atrial tachycardia are often likely to be a nonspecific response to atrial stimulation, for the purposes of the present study, inducible atrial tachycardia was defined as a monomorphic atrial tachycardia that had cycle length $<600 \mathrm{msec}$ and duration $>5$ seconds, and was reproducibly inducible (Fig. 1). The following criteria were used to diagnose atrial tachycardia: (1) induction by an atrial depolarization that blocked in the AV node; (2) induction independent of a critical atrial-His interval; (3) eccentric atrial activation; (4) a variable VA relationship during tachycardia; (5) failure to terminate the tachycardia by ventricular pacing without advancing the atrial electrograms; (6) a "V-A-A" response upon cessation of pacing after entrainment with ventricular pacing ${ }^{1}$; and (7) when the tachycardia was nonsustained, consistent spontaneous termination in the absence of AV block. The atrial tachycardia was considered sustained if its duration was $>30$ seconds.

None of 27 patients who had inducible atrial tachycardia initially were treated for the tachycardia with either radiofrequency catheter ablation or antiarrhythmic drug therapy. However, $6(22 \%)$ of these patients were treated with verapamil, atenolol, or flecainide for hypertension, inappropriate sinus tachycardia, or paroxysmal atrial fibrillation.

The patients were seen in an outpatient clinic 4 months after the electrophysiologic procedure and were told to contact one of the authors in the event of a recurrence of palpitations. In the event of recurrent symptoms, the patient was provided with a continuous-loop event monitor to document the rhythm at the time of symptoms. Mean duration of follow-up was $9.7 \pm 5.8$ months (range 1.1 to 19 ).

\section{Statistical Analysis}

Continuous variables are expressed as mean $\pm 1 \mathrm{SD}$. Comparisons were performed with Student's $t$-test or the Fisher exact test. $\mathrm{P}<0.05$ was considered significant.

\section{Results}

\section{Prevalence of Induced Atrial Tachycardia}

Among the 176 patients, 27 patients (15\%) had inducible atrial tachycardia. Age, gender, ejection fraction, left atrial diameter, and prevalence of structural heart disease did not differ significantly between patients with and patients without inducible atrial tachycardia (Table 1). 
TABLE 1

Clinical Characteristics of Patients With and Without Inducible Atrial Tachycardia

\begin{tabular}{lccc}
\hline & $\begin{array}{c}\text { Inducible } \\
\text { Atrial } \\
\text { Tachycardia }\end{array}$ & $\begin{array}{c}\text { No Inducible } \\
\text { Atrial } \\
\text { Tachycardia }\end{array}$ & P Value \\
\hline No. of patients & $27(15 \%)$ & $149(85 \%)$ & \\
Age (years) & $49 \pm 15$ & $48 \pm 18$ & 0.9 \\
Ejection fraction & $0.59 \pm 0.03$ & $0.58 \pm 0.06$ & 0.3 \\
Left atrial diameter (mm) & $36 \pm 5$ & $36 \pm 7$ & 0.8 \\
Female gender & $21(78 \%)$ & $109(73 \%)$ & 0.4 \\
Heart disease & $4 / 27(15 \%)$ & $22 / 149(15 \%)$ & 0.6 \\
\hline
\end{tabular}

Continuous values are expressed as mean \pm SD.

\section{Characteristics of Induced Atrial Tachycardia}

Among the 27 patients with inducible atrial tachycardia, the tachycardia was nonsustained in 20 patients (74\%) and was inducible only during isoproterenol infusion in 20 patients (74\%). Mean tachycardia cycle length was $350 \pm 117$ msec for the sustained episodes and $352 \pm 89 \mathrm{msec}$ for the nonsustained episodes $(\mathrm{P}=1.0)$. The sustained episodes had a mean duration of $107 \pm 96$ seconds, and the nonsustained episodes had a mean duration of $10 \pm 8$ seconds $(\mathrm{P}<$ $0.001)$. The sustained episodes were terminated by atrial overdrive pacing in 2 of 7 patients and terminated spontaneously after 30 seconds in the remaining patients.

Atrial tachycardia was induced by right atrial overdrive pacing in 8 patients, right ventricular overdrive pacing in 2 , programmed atrial stimulation with a single extrastimulus in 11, programmed atrial stimulation with two extrastimuli in 5 , and programmed ventricular stimulation with a single extrastimulus in 1 .

\section{Recurrent Palpitations}

Six $(22 \%)$ of the 27 patients with inducible atrial tachycardia had recurrent symptoms of palpitations after successful ablation of AV nodal reentrant tachycardia. The palpitations occurred at $108 \pm 123$ days of follow-up (range 14 to 350 ) and were described as being similar to the palpitations experienced before the ablation procedure by 1 patient and as different from the palpitations experienced before the ablation procedure by 5 . Four patients had palpitations lasting $>30$ seconds, and 2 patients had episodes lasting $<30$ seconds.

ECG documentation with a continuous-loop event recorder demonstrated a probable atrial tachycardia in two patients (sustained in one and nonsustained in the other) (Fig. 2). In both cases, the documented atrial tachycardia had a cycle length similar to the induced episode (540 vs $480 \mathrm{msec}$, and $440 \mathrm{vs} 460 \mathrm{msec}$ ). One patient had a recording consistent with AV nodal reentrant tachycardia (confirmed by repeat electrophysiologic procedure), 1 had inappropriate sinus tachycardia, 1 had paroxysmal atrial fibrillation, and 1 had frequent premature atrial complexes (Table 2).

Among the 27 patients with inducible atrial tachycardia, the tachycardia was sustained and inducible in the absence of isoproterenol in 4 patients. These characteristics were not predictive of recurrent palpitations after slow pathway ablation. Three of these 4 patients remained asymptomatic during follow-up, and only 1 (25\%) experienced symptom- atic sustained atrial tachycardia during follow-up. Among the 23 patients in whom inducible atrial tachycardia was not sustained and isoproterenol dependent, 1 patient (4\%) experienced symptomatic atrial tachycardia (nonsustained) during follow-up $(P=0.3)$. The patient with sustained atrial tachycardia was treated with propafenone, and the patient with nonsustained atrial tachycardia was treated with atenolol. Both patients have remained asymptomatic with this therapy.

\section{Discussion}

\section{Main Findings}

The results of this study demonstrate that atrial tachycardia may be inducible in $15 \%$ of patients who undergo radiofrequency ablation of AV nodal reentrant tachycardia and who do not have prior clinical documentation of atrial tachycardia. The majority of the episodes of inducible atrial tachycardia induced in this study were nonsustained and isoproterenol dependent. Fewer than $10 \%$ of the patients in whom atrial tachycardia was induced later experienced symptoms attributable to atrial tachycardia. This observation suggests that inducible atrial tachycardia, even when monomorphic and reproducibly inducible, often may be a nonspecific finding that does not have clinical significance. Therefore, when atrial tachycardia is inducible in a patient with AV nodal reentrant tachycardia who does not have documentation of prior atrial tachycardia, therapy directed at the atrial tachycardia should be deferred and limited to the occasional patient who later develops symptomatic atrial tachycardia.

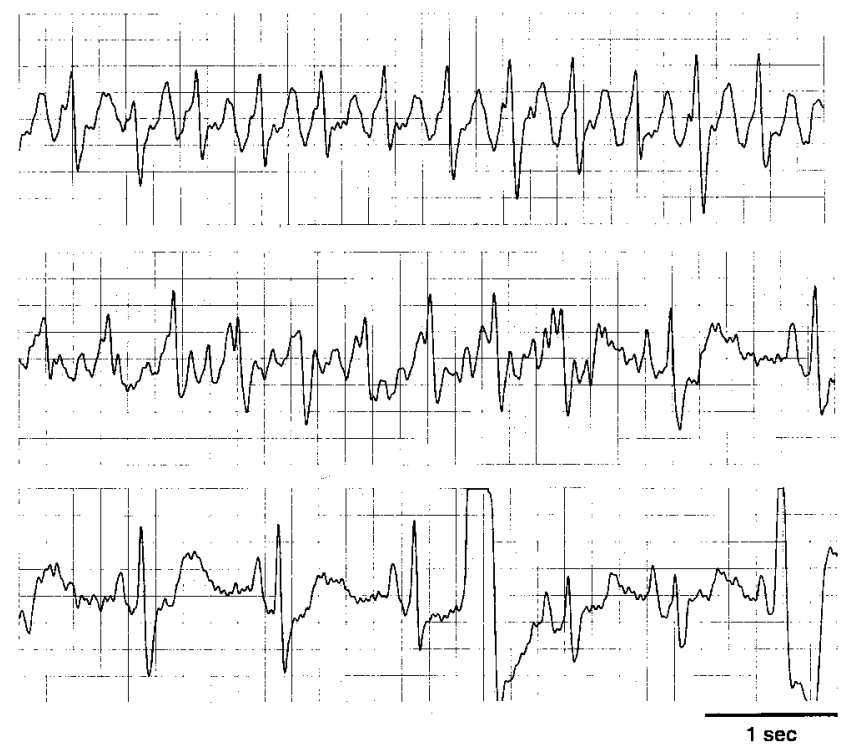

Figure 2. Recording obtained with a continuous-loop event recorder at the time of palpitations 20 days after successful slow pathway ablation in the same patient who had the inducible atrial tachycardia shown in Figure 1. The cycle length of this tachycardia is $480 \mathrm{msec}$, and the tracing is suggestive of an atrial tachycardia. The recordings are continuous, with the top strip displaying probable atrial tachycardia, the middle strip showing spontaneous conversion to sinus rhythm, and the bottom strip showing the artifact caused by activation of the recorder by the patient. 
TABLE 2

Clinical Features of the Six Patients with Inducible Atrial Tachycardia who Experienced Palpitations after Successful Slow Pathway Ablation

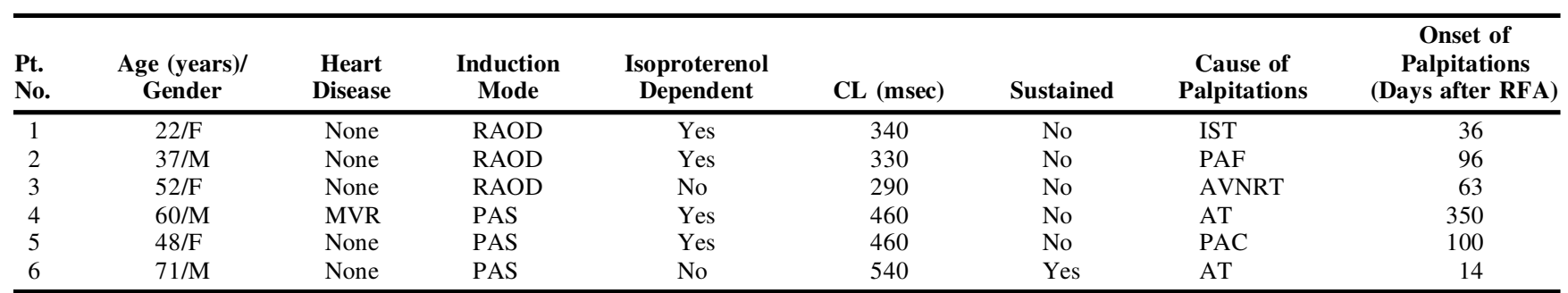

AT $=$ atrial tachycardia; AVNRT $=$ AV nodal reentrant tachycardia; $\mathrm{CL}=$ cycle length; $\mathrm{IST}=$ inappropriate sinus tachycardia; $\mathrm{MVR}=$ mitral valve repair; $\mathrm{PAC}=$ premature atrial complex; $\mathrm{PAF}=$ paroxysmal atrial fibrillation; PAS $=$ programmed atrial stimulation; RAOD $=$ right atrial overdrive pacing; RFA $=$ radiofrequency ablation.

\section{Tachycardia Substrate}

AV nodal reentrant tachycardia and accessory pathway tachycardias have a clearly defined anatomic substrate that gives rise to reentrant tachycardias and are amenable to radiofrequency catheter ablation. ${ }^{2}$ However, in the case of atrial tachycardia, the underlying tachycardia mechanism may be reentry, abnormal automaticity, or triggered activity. ${ }^{3}$ The atrial tachycardias induced in the present study were inducible by pacing maneuvers, consistent with either reentry or triggered activity. These types of atrial tachycardia may not require a specific anatomic substrate, which explains why they may often represent a nonspecific finding.

\section{Previous Studies}

Previous studies showed that symptomatic palpitations after slow pathway ablation for AV nodal reentrant tachycardia, or after accessory pathway ablation, occur in $32 \%$ to $36 \%$ of patients. ${ }^{4,5}$ Atrial tachycardia was found to be the cause of recurrent palpitations in $<5 \%$ of the patients who had recurrent symptoms. The low incidence of recurrent palpitations attributable to atrial tachycardia after catheter ablation of AV nodal reentrant tachycardia or of an accessory pathway in these two prior studies is consistent with the results of the present study.

In this series, 7 (26\%) of the 27 patients who underwent successful slow pathway ablation were treated during follow-up with an antiarrhythmic drug for paroxysmal atrial fibrillation, atrial tachycardia, or inappropriate sinus tachycardia. This is similar to the results of a prior study in which $17 \%$ of 398 patients who underwent successful accessory pathway ablation were treated with an antiarrhythmic medication during follow-up. ${ }^{6}$

\section{Limitations}

Some of the patients who had inducible atrial tachycardia may have had asymptomatic recurrences of atrial tachycardia during follow-up. Therefore, the predictive value of inducible atrial tachycardia may have been underestimated. However, if episodes of atrial tachycardia during follow-up are asymptomatic, therapy is not necessary and the results of this study remain valid for clinically significant episodes of atrial tachycardia.

A second limitation is that six patients were treated with an antiarrhythmic drug for hypertension or for an arrhyth- mia other than atrial tachycardia. It is possible that drug therapy in these patients suppressed symptomatic atrial tachycardia that otherwise may have occurred.

\section{Conclusion}

Radiofrequency catheter ablation of atrial tachycardia has a lower success rate than catheter ablation of AV nodal reentrant tachycardia or accessory pathways, ${ }^{2}$ a higher recurrence rate after ablation (5\% to $18 \%$ ), ${ }^{7,8}$ and a higher potential for multifocality. ${ }^{2}$ Furthermore, mapping of atrial tachycardias is tedious when the episodes of induced tachycardia are nonsustained. ${ }^{9}$ For these reasons, and because the majority of patients with inducible atrial tachycardia after slow pathway ablation remain asymptomatic, we recommend deferring catheter ablation or drug therapy for the inducible atrial tachycardia, unless there is documentation that the atrial tachycardia is clinically significant.

\section{References}

1. Knight BP, Zivin A, Souza J, Flemming M, Pelosi F, Goyal R, Man KC, Strickberger SA, Morady F: A technique for the rapid diagnosis of atrial tachycardia in the electrophysiology laboratory. J Am Coll Cardiol 1999;33:775-781.

2. Morady F: Radio-frequency ablation as treatment for cardiac arrhythmias. N Engl J Med 1999;340:534-544.

3. Steinbeck G, Hoffmann E: "True" atrial tachycardia. Eur Heart J 1998;19:E10-E12.

4. Jordaens L, Vertongen P, Verstraten T: Prolonged monitoring for detection of symptomatic arrhythmias after slow pathway ablation in AV-nodal tachycardia. Int J Cardiol 1994;44:57-63.

5. Mann DE, Kelly PA, Adler SW, Fuenzalida CE, Reiter MJ: Palpitations occur frequently following radiofrequency catheter ablation for supraventricular tachycardia, but do not predict pathway recurrence. PACE 1993;16:1645-1649.

6. Dagres N, Clague JR, Kottkamp H, Hindricks G, Breithardt G, Borggrefe M: Radiofrequency catheter ablation of accessory pathways: Outcome and use of antiarrhythmic drugs during follow-up. Eur Heart J 1999;20:1826-1832.

7. Chen SA, Chiang CE, Yang CJ, Cheng CC, Wu TJ, Wang SP, Chiang BN, Chang MS: Sustained atrial tachycardia in adult patients. Electrophysiological characteristics, pharmacological response, possible mechanisms, and effects of radiofrequency ablation. Circulation 1994; 90:1576-1577.

8. Kay GN, Chong F, Epstein AE, Dailey SM, Plumb VJ: Radiofrequency ablation for treatment of primary atrial tachycardias. J Am Coll Cardiol 1993;21:901-909.

9. Lesh MD, Van Hare GF, Epstein LM, Fitzpatrick AP, Scheinman MM, Lee RJ, Kwasman MA, Grogin HR, Griffin JC: Radiofrequency catheter ablation of atrial arrhythmias. Results and mechanisms. Circulation 1994;89:1074-1089. 